RESEARCH ARTICLE

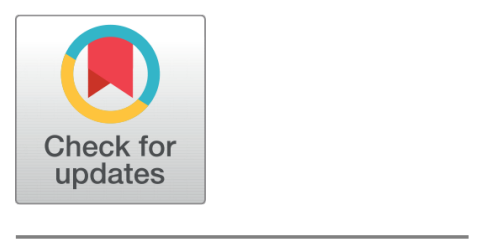

OPEn ACCESS

Received: 06.06.2021

Accepted: 29.08.2021

Published: 27.09 .2021

Citation: Reddy DN, Ravinder Y (2021) Spectrum Sensing in Non-Gaussian Noise. Indian Journal of Science and Technology 14(32): 2596-2606. https://doi.org/ 10.17485/IJST/v14i32.1034

* Corresponding author.

reddydeepa2680@gmail.com

Funding: None

Competing Interests: None

Copyright: ( $) 2021$ Reddy \& Ravinder. This is an open access article distributed under the terms of the Creative Commons Attribution License, which permits unrestricted use, distribution, and reproduction in any medium, provided the original author and source are credited.

Published By Indian Society for Education and Environment (iSee)

ISSN

Print: 0974-6846

Electronic: 0974-5645

\section{Spectrum Sensing in Non-Gaussian Noise}

\author{
Deepa N Reddy ${ }^{1}$, Yerram Ravinder ${ }^{2}$ \\ 1 Assistant Professor, Department of Electronics and Communication, BMS Institute of \\ Technology \& Management, Bengaluru, India \\ 2 Professor, Department of Electronics and Telecommunication, Pune Institute of Computer \\ Technology, Pune, India
}

\section{Abstract}

Background: Spectrum sensing is a crucial step to realize the Cognitive Radio technology. The spectrum sensing schemes at low signal-to-noise ratio, noise uncertainty and especially under the background of non-Gaussian noise, provide low detection of the primary user. This results in missed detection or false alarm and increases higher interference to the primary user. Objectives: Detection schemes designed for additive Gaussian noise exhibit poor performance in the non-Gaussian environment. This study considers the problem of spectrum sensing with the assumption that the noise follows a non-Gaussian distribution with heavier tails. Methods/findings: A fuzzy logicbased method is proposed for primary user detection under non Gaussian Noise. The results are highlighted for the Laplacian noise. Through Monte Carlo simulations it is observed that Laplacian noise noticeably affects the performance of energy detector. Also, a fractional change in noise uncertainty degrades the performance of energy detector. The performance of the proposed scheme is presented through receiver operating characteristic (ROC) and plot of the detection probability versus signal-to-noise ratio (SNR) using simulations. It is shown that by appropriately choosing the membership functions and the fuzzy rule base in the fuzzy inference system the proposed fuzzy logic method for spectrum sensing provides reliable detection.

Keywords: NonGaussian noise; Fuzzy logic; Spectrum sensing; noise uncertainty

\section{Introduction}

Majority of spectrum sensing schemes are focused on primary user detection in Gaussian noise. The Gaussian distribution fails to satisfactorily describe some noise in practice. For instance, the Gaussian distribution cannot be used to model Radio Frequency $(\mathrm{RF})$ noise and low frequency atmospheric noise. This is primarily due to the fact that the noise in practice is likely to generate observations of high magnitude than what can be produced from the Gaussian distribution. High magnitude observations are also referred to as impulsive noise. This conveys that the probability distribution function (pdf) of such noise has heavy tails. It can be stated that the pdf of the 
non-Gaussian impulsive noise decays at a lower rate than the Gaussian. Therefore, a prime feature of the non-Gaussian model is that it has heavy tails than the Gaussian distribution. Also in practical wireless communication scenarios the noise usually has a heavy tailed nature. The Laplacian noise is an important non-Gaussian noise distribution and hence it is frequently used in engineering studies.

For accurate detection of primary user (PU) signal under noise uncertainty many detection schemes are designed for additive Gaussian noise. The effect of threshold selection over the performance of spectrum sensing in cognitive radio network (CRN) using energy detector (ED) is studied in ${ }^{(1-4)}$. Through simulations it is shown that it provides better detection performance through suitable selection of the dynamic threshold factor. The effect of noise uncertainty on the energy detection approach is analyzed in ${ }^{(5,6)}$. $\operatorname{In}^{(6)}$ a two-step cooperative stochastic resonance energy detection algorithm is proposed to address the problem, where the traditional energy detection (ED) performance is susceptible to noise uncertainty.

Recently, under the category of non-parametric detection, some detection schemes based on the Goodness-of-fit test (GoFT) have been proposed. They include the Anderson-Darling (AD) detection, the Kolmogorov-Smirnov (KS) test, the detection based on ordered statistics and so on. These schemes perform better than energy detection in a Gaussian environment ${ }^{(7)}$. A Frequency domain Goodness of Fit Test based spectrum sensing method is proposed under dynamically varying noise. Through analytical and simulated results, it is shown that this method is robust to dynamically varying noise ${ }^{(8)}$. In ${ }^{(9-11)}$ fuzzy logic is used to address the decision on the PU activity. This scheme showed better detection performance in low signal to noise ratio (SNR) regions. It is also shown through simulation results that the fuzzy based detection could achieve accurate sensing results better than conventional schemes in noisy conditions. Several studies in ${ }^{(12-15)}$ have focused on signal detection under a NGN environment. Schemes like energy detection, sequential detection and absolute value cumulation detection are investigated in a Laplacian environment. However, there still exist some issues in PU detection in Cognitive Radio (CR). Two primary issues are 1) Improving the detector's performance under non-Gaussian noise, 2) need for computationally efficient method to meet the real-time detection requirement.

This work considers the problem of spectrum sensing with the assumption that the noise follows a non-Gaussian distribution with heavier tails. The main contributions in this paper are summarized as follows:

- The effect of non-Gaussian noise on the energy detector's performance in noise uncertain environment is investigated.

- To overcome the effect of noise uncertainty, the spectrum sensing algorithm using adaptive fuzzy threshold proposed in ${ }^{(16)}$ primarily studied under Gaussian noise is investigated under non-Gaussian noise environment.

- The effect on the probability of detection due to the characterization of the statistical moments in the noise distribution is investigated.

- Performance evaluation of the conventional and fuzzy threshold methods are carried out through simulations under different noise uncertainty conditions.

- The best method out of these techniques under varying noise uncertainty conditions is identified.

The rest of this paper is organized as follows. Section 2 gives the outline on the non-Gaussian noises. Section 3 and 4 detail the system model for the spectrum sensing and the proposed method. The results are presented and discussed in Section 5 and concluding remarks are provided in Section 6.

\section{Non-Gaussian Noise}

Pearson introduced kurtosis to understand whether a given distribution is Gaussian or not. Three conditions proposed by Pearson for a distribution to be called as Gaussian are (i) kurtosis, (ii) skewness, (iii) the distance between mean and mode (modal divergence). Skewness and kurtosis are the higher order moments that are used to determine the difference between other distributions and the Gaussian distribution ${ }^{(17,18)}$.

Since the Laplace distribution is the most popular distribution and is frequently used in engineering studies this work focuses on primary user signal detection in Laplacian noise. The Laplace distribution is within the class of generalized Gaussian distributions.

The Gaussian pdf is given as

$$
p(n)=\frac{1}{\sqrt{2 \pi \sigma^{2}}} \exp \left(-\frac{n^{2}}{2 \sigma^{2}}\right)
$$

The Laplacian pdf is given by

$$
p(n)=\frac{1}{\sqrt{2 \sigma^{2}}} \exp \left(-\sqrt{\frac{2}{\sigma^{2}}}|n|\right),-\infty<n<\infty
$$


Where, $\sigma^{2}$ is the noise variance

Normally, a heavier tailed noise has a larger degree of non-Gaussianity, also known as kurtosis, defined as

$$
k=\frac{E\left[n^{4}\right]}{E^{2}\left[n^{2}\right]}
$$

Equivalently the kurtosis coefficient minus 3 is referred to as excess kurtosis. As the kurtosis increases the tails are heavier and $\mathrm{k}$ as 6 (excess kurtosis is 3) corresponds to Laplacian noise, while when the pdf is Gaussian the excess kurtosis is 0 . Figure 1 gives the pdf plot of Eqn (1) and (2). It is observed that the non-Gaussian model has heavier tail than the Gaussian distribution.

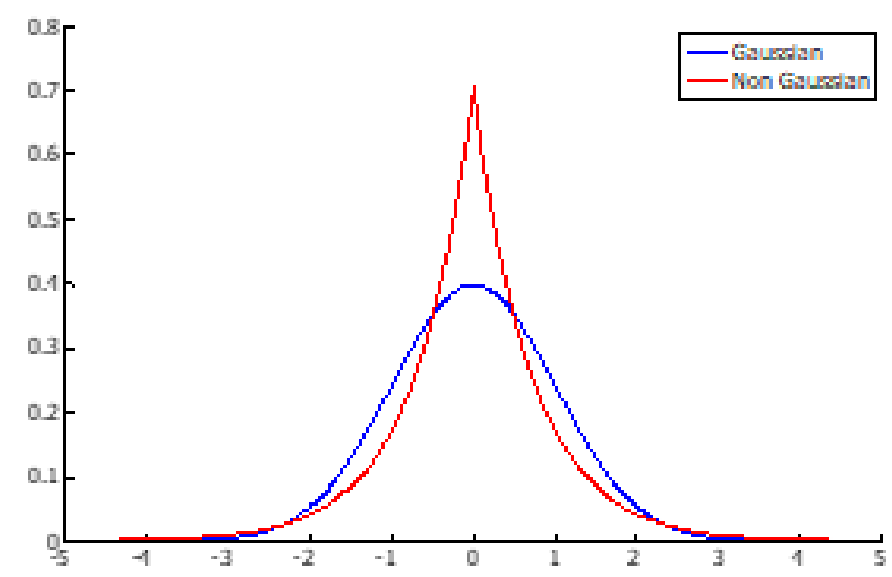

Fig 1. Gaussian and non Gaussian distributions

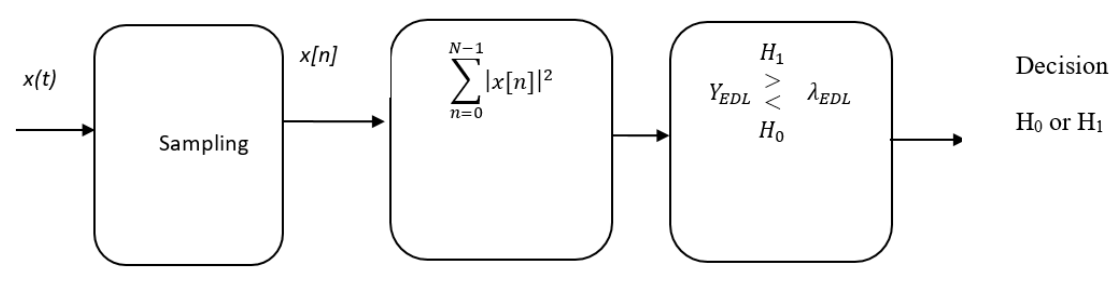

Fig 2. Energy Detector under Laplacian noise

\section{System Model}

The detection of the PU activity in the presence of noise by the secondary user (SU) is modeled as a binary hypothesis testing problem as given below:

$$
\left\{\begin{array}{c}
H_{0}: x[n]=v[n] \\
H_{1}: x[n]=s[n]+v[n]
\end{array}\right\}
$$

where $\mathrm{v}[\mathrm{n}]$ 's are independent and identically distributed (i.i.d.) noises, with non-Gaussian distribution of zero mean and variance $\sigma_{w}^{2}$ given as $\mathrm{L}\left(0, \sigma_{w}^{2}\right)$ and $\mathrm{s}[\mathrm{n}]$ is the signal transmitted by the PU.

\subsection{Energy Detector under Laplacian Noise}

The energy detector (ED) is the most commonly used method for PU detection as it has very low computational complexity. The energy detector under Laplacian noise (EDL) is discussed in ${ }^{(19)}$. The block diagram of energy detector under Laplacian noise is given in Figure 2. 
The test statistic of energy detector under Laplacian noise is given as

$$
Y_{E D L}=\sum_{n=1}^{N}|x[n]|^{2} \stackrel{H_{1}}{>} \underset{ }{>} \lambda_{E D L}
$$

The energy measurement $Y_{E D L}$ is calculated from $\mathrm{N}$ samples of the received signal at the $\mathrm{CR}$ receiver. The decision static $Y_{E D L}$ is compared against a fixed threshold $\lambda_{E D L}$. The simple hypothesis testing problem is formulated in Eqn (5), where $\lambda_{E D L}$ is the detection threshold which is set assuming constant false alarm rate. The sensing performance of the energy detector in Laplacian noise is measured by two metrics called as the detection probability $\left(\mathrm{P}_{D}\right)$ and false alarm probability $\left(\mathrm{P}_{F}\right)$ as formulated in ${ }^{(19)}$.

$$
\begin{gathered}
P_{F}=Q\left(\frac{\lambda_{E D L}-N \sigma_{w}^{2}}{\sqrt{5 N} \sigma_{w}^{2}}\right) \\
P_{D}=Q\left(\frac{\lambda_{E D L}-N\left(P+\sigma_{w}^{2}\right)}{\left.\sqrt{5 N \sigma_{w}^{4}+4 N P \sigma_{w}^{2}}\right)}\right)
\end{gathered}
$$

Where, $\mathrm{P}$ gives the power of the $\mathrm{PU}$ signal, $\mathrm{N}$ gives the number of samples, variance $\sigma_{w}^{2}, \lambda_{E D L}$ is the detection threshold and $\mathrm{Q}($.$) is the Gaussian complementary CDF.$

\subsection{Effect of Noise Uncertainty on Energy Detector under Laplacian Noise}

In practical scenarios, it is not possible to accurately know the statistics of the noise $v[n]$. This creates the uncertainty in noise in the detection that may vary with time. The noise uncertainty is modelled such that the noise power is distributed in a single interval as given below.

$$
\sigma_{w}^{2} \varepsilon\left[\left(\frac{1}{\rho}\right) \sigma_{n}^{2}, \rho \sigma_{n}^{2}\right]
$$

Where, $\sigma_{n}^{2}$ is the nominal noise power and $\rho>1$ indicates the uncertainty quantification.

The SNR wall for an energy detector under Laplacian noise is given in ${ }^{(19,20)}$. It is the SNR beneath which reliable signal detection cannot be achieved.

$$
S N R_{\text {wall }}^{L E D}=\frac{\rho^{2}-1}{\rho}
$$

Considering the worst-case noise uncertainty Eqn (6) and (7) which gives false alarm and the probability of detection respectively are modified.

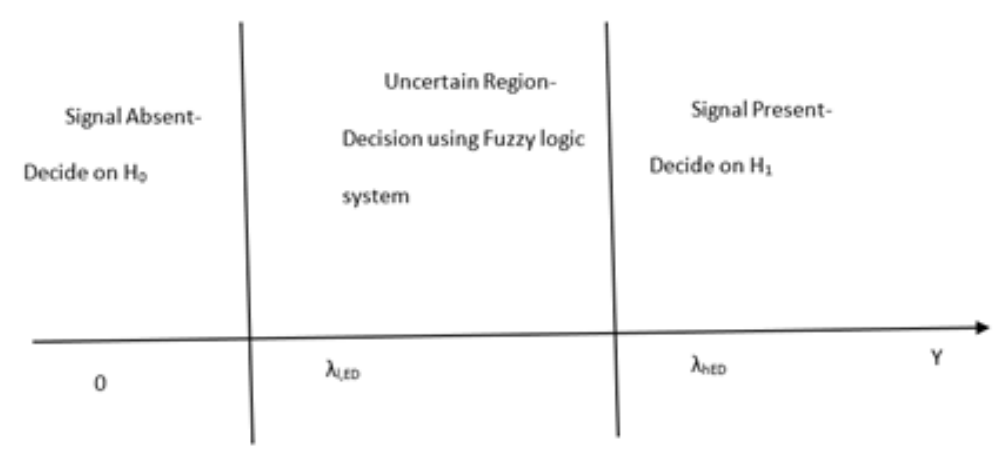

Fig 3. Two thresholds of the energy detector under Laplacian Noise 


$$
\begin{gathered}
P_{F}^{\prime}=Q\left(\frac{\lambda_{E D L}-N \rho \sigma_{w}^{2}}{\sqrt{5 N} \rho \sigma_{w}^{2}}\right) \\
P_{D}^{\prime}=Q\left(\frac{\left.\lambda_{E D L}-N\left(P+\frac{\left(\sigma_{w}^{2}\right.}{\rho}\right)\right)}{\left.\sqrt{5 N \sigma_{w}^{4}(1 / \rho)+4 N P(1 / \rho) \sigma_{w}^{2}}\right)}\right)
\end{gathered}
$$

The decision on PU activity by the CR device is usually made by a conventional ED using a single threshold. But for accurate detection it is necessary to fix an optimum threshold level, as the detection performance is dependent on the threshold level. The optimum threshold cannot be easily defined due to the problem of noise uncertainty. The situation worsens with low SNR, since the noise uncertainty leads to SNR Wall which is defined as the SNR below which the detector cannot provide reliable detection. In addition, the detection performance is dissatisfactory under the non-Gaussian noise in comparison to the Gaussian noise ${ }^{(19,20)}$.

\section{Proposed Method}

To overcome the effect of noise uncertainty in CR systems several studies have been proposed as discussed in Section 1 of the paper. In this study a fuzzy thresholding scheme is investigated under non-Gaussian scenarios. This study is the first of its kind which uses a fuzzy thresholding approach for PU detection in a non-Gaussian environment. The two thresholds are therefore formulated from Eqn (6) as

$$
\begin{gathered}
\lambda_{l, E D L}=\left(\sqrt{5 N} Q^{-1}\left(P_{F}\right)+N\right)\left(\frac{1}{\rho}\right) \sigma_{w}^{2} \\
\lambda_{h, E D L}=\left(\sqrt{5 N} Q^{-1}\left(P_{F}\right)+N\right) \rho \sigma_{w}^{2}
\end{gathered}
$$

Where, $\lambda_{l, E D L}$ is the lower threshold and $\lambda_{h, E D L}$ is the upper threshold. These thresholds are pictorially represented in Figure 3. Using these two thresholds the whole range of the observed test statistic $Y_{E D L}$ is divided into three zones 1) Signal present zone $-\mathrm{H}_{1}$ declared 2) uncertainty zone and 3) Signal absent zone $-\mathrm{H}_{0}$ declared. If the test statistic exceeds the upper threshold $\lambda_{h, E D L}$ then the $\mathrm{SU}$ decides on $\mathrm{H}_{1}$ which means the presence of primary user. While if the test statistic is less than $\lambda_{l, E D L}$ the $\mathrm{SU}$ decides on $\mathrm{H}_{0}$. The lower and upper thresholds are selected in accordance with the lower and the upper limit of noise variance respectively.

The decision process using the two thresholding scheme is given below:

$$
D=\left\{\begin{array}{cccl}
1 & \text { if } & Y>\lambda_{h, E D L} & H_{1} \text { is declared } \\
F & \text { if } & \lambda_{l, E D L}<Y<\lambda_{h, E D L} & \text { Fuzzy decision } \\
0 & \text { if } & Y>\lambda_{l, E D L} & H_{0} \text { is declared }
\end{array}\right\}
$$

The fuzzy decision F is defined as

$$
F=\left\{\begin{array}{ll}
1 \text { if } Z>\lambda_{F}, & H_{1} \text { is declared } \\
0 \text { if } Z<\lambda_{F}, & H_{0} \text { is declared }
\end{array}\right\}
$$




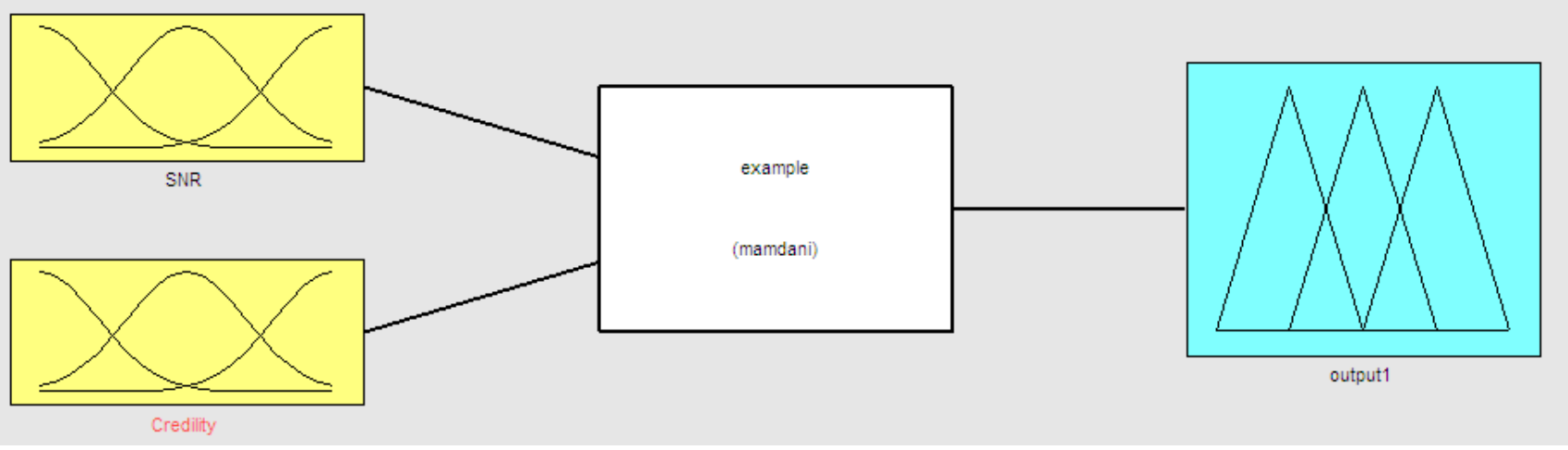

Fig 4. Membership functions in the input and output of the fuzzy inference system

Where, $\mathrm{Z}$ is the output of the fuzzy logic system, $\lambda_{F}$ is the threshold set for taking fuzzy logic decision.

The final decision $\mathrm{X}=\mathrm{D}+\mathrm{F}$

$$
X=\left\{\begin{array}{cc}
D+F>0, & P U \text { is present } \\
\text { else, } & \text { PU is absent }
\end{array}\right\}
$$

The decision in the confused region is taken using two antecedents called 1) Credibility 2) SNR available at SU. The output of the fuzzy logic system is the consequent which gives the possibility of the primary user activity.

The credibility $\mathrm{C}$ is defined as

$$
C=\frac{\left(Y_{E D L}-\lambda_{l, E D L}\right)}{\left(\lambda_{h, E D L}-\lambda_{l, E D L}\right)}
$$

If the value of $\mathrm{C}$ is high, then $\mathrm{H}_{1}$ is favoured and if the value of $\mathrm{C}$ is low $\mathrm{H}_{0}$ is favoured.

The fuzzy rule base and the membership for the antecedents and consequents are detailed in Figure 4 and Table 1 respectively.

Table 1. Rule base for decision on the PU activity

\begin{tabular}{lll}
\hline $\begin{array}{l}\text { Antecedent } 1 \\
\text { (Credibility) }\end{array}$ & $\begin{array}{l}\text { Antecedent } 2 \\
\text { (SNR) }\end{array}$ & $\begin{array}{l}\text { Consequent } \\
\text { (Likelihood of the presence of PU) }\end{array}$ \\
\hline Low & Low & Very Low \\
Low & Medium & Low \\
Low & High & Medium \\
Medium & Low & Low \\
Medium & Medium & Medium \\
Medium & High & High \\
High & Low & Medium \\
High & Medium & High \\
High & High & Very High \\
\hline
\end{tabular}

\section{Results and Discussion}

This section details the results obtained through MATLAB simulations to analyze the performance of fuzzy two threshold scheme under non-Gaussian noise. 


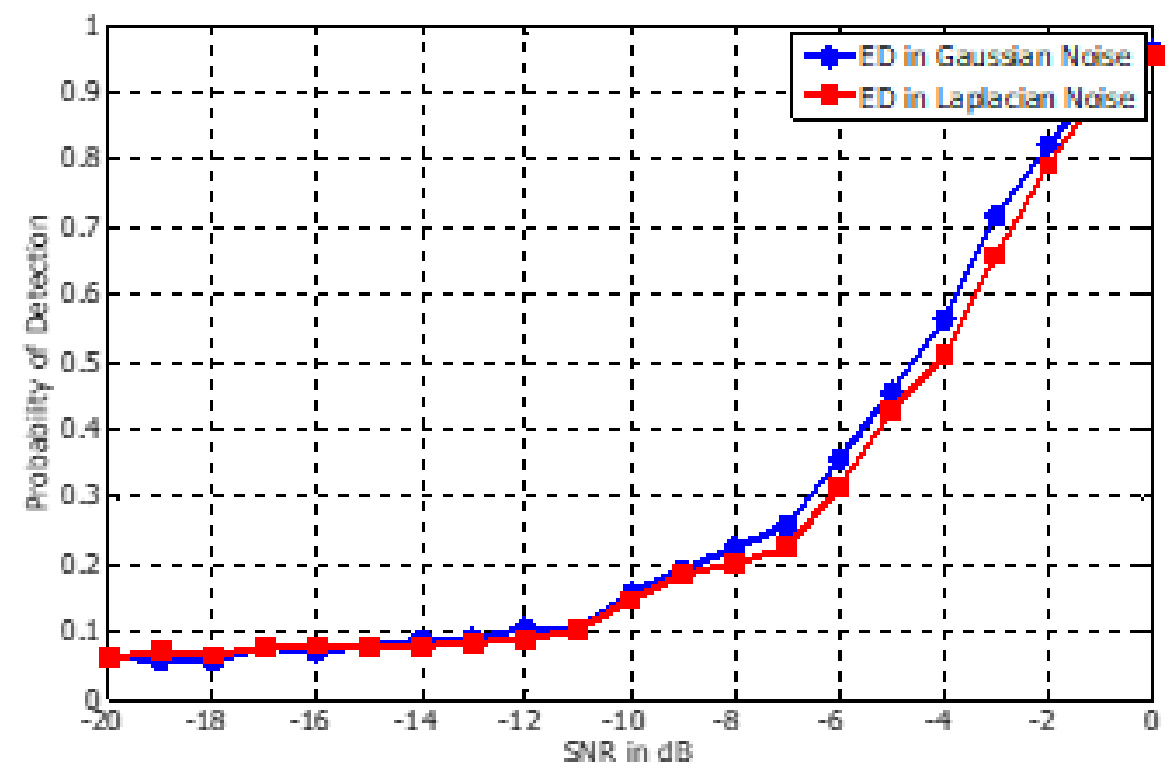

Fig 5. Performance of energy detector under Gaussian and non-Gaussian noise

The spectrum sensing is carried out in a CR environment using energy detection. The detection probability is used as a standard of measurement to determine the sensing accuracy. The primary transmitter signal is considered as a sinusoidal pilot signal of known frequency. The noise is modeled as non-Gaussian. In the simulations the Laplacian noise is considered, as it is a special case of non-Gaussian noise. It has pdf with excess kurtosis of 3 while the pdf of the Gaussian has the excess kurtosis as $0^{(17)}$. The results provided are averaged over 10000 Monte Carlo simulations. Table 2 outlines the parameters used in the simulation study.

Figure 5 gives the performance of ED under the Gaussian and non-Gaussian noise. From the plot it is evident that the energy detector performs better under Gaussian noise than Laplacian noise. The energy detector provides optimal performance under Gaussian noise even when no prior information on the PU signal is available. Hence it is necessary to investigate methods to boost the energy detector's performance under Laplacian noise.

Table 2. Values used in Simulations

\begin{tabular}{lll}
\hline Parameters & & Values \\
\hline Target false alarm probability $\overline{P_{F}}$ & 0.05 \\
Total numbers of samples N & & 100 \\
SNR range of interest & Gaussian & -20 to $-5 \mathrm{~dB}$ \\
Excess Kurtosis & Non-Gaussian & 0 \\
& & 3 \\
\hline
\end{tabular}

Figure 6 illustrates the impact of noise uncertainty on the energy detector's performance under Laplacian noise. Energy detection using single threshold in the detection process is sensitive to noise uncertainty. A marginal change in the noise uncertainty factor $\rho$ deteriorates the performance quickly. 


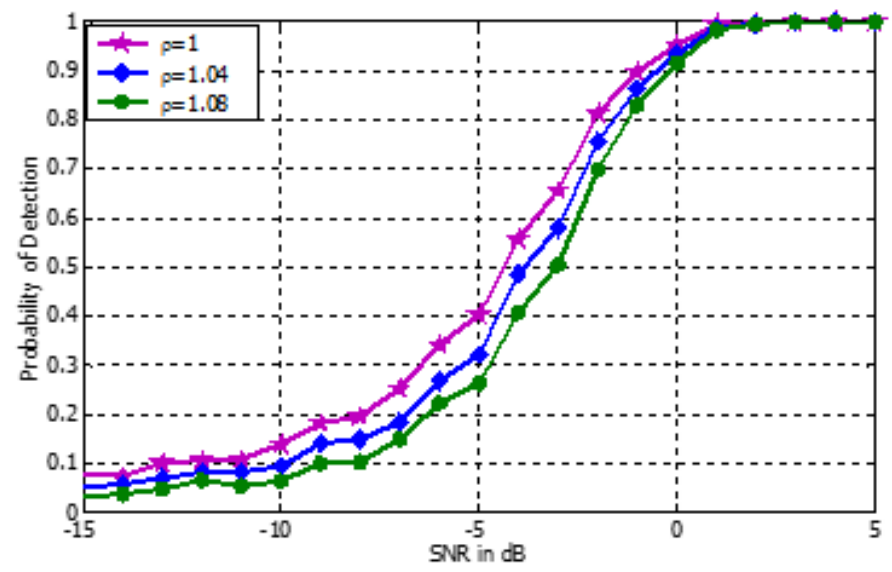

Fig 6. Impact of noise uncertainty on detection probability under non-Gaussian noise

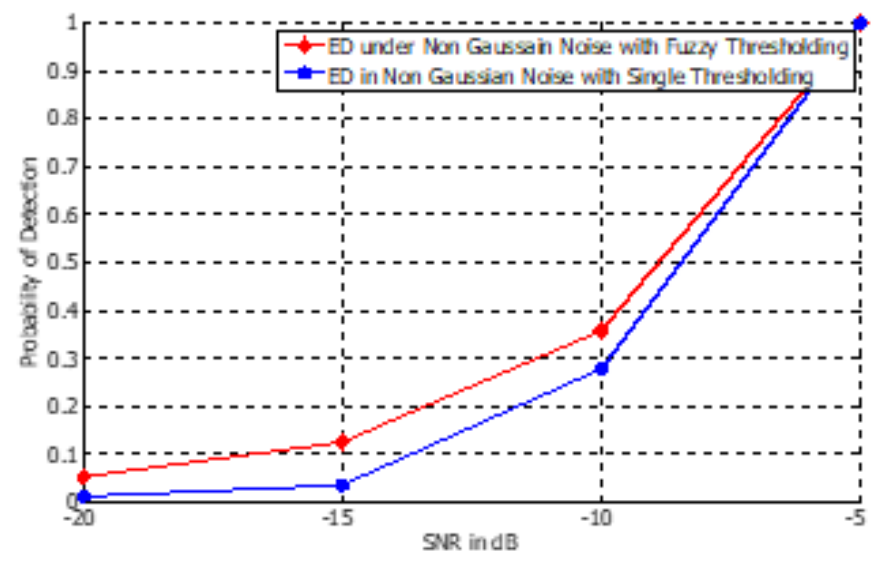

Fig 7. Effect of Energy detector under non-Gaussian Noise for $\rho=1.023, \mathrm{P}_{F}=0.01, \mathrm{~N}=1000$ Samples

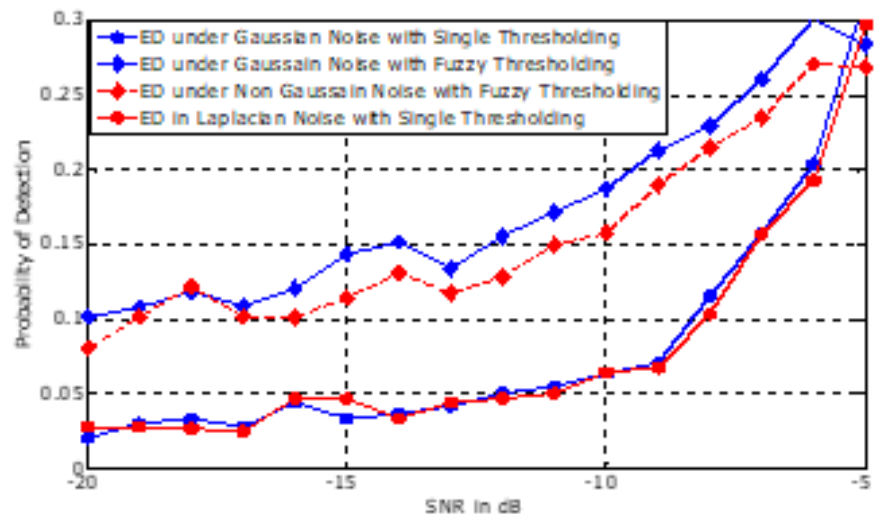

Fig 8. Thresholding methods to combat noise uncertainty in detection performance for $\rho=1.088$ 
The method proposed in ${ }^{(16)}$ to address the problem of noise uncertainty are simulated and compared with the single threshold using the constant false alarm rate. From Figures 7 and 8 , it is observed that the PU detection using single threshold fails as the noise uncertainty increases, because the threshold fixed is independent of the noise variance.

Table 3. Results obtained using Single and Fuzzy thresholding schemes

\begin{tabular}{lllll}
\hline SNR & $\begin{array}{l}\text { PD using Single Threshold- } \\
\text { ing under Gaussian Noise }\end{array}$ & $\begin{array}{l}\text { PD using Single Thresholding } \\
\text { under Non Gaussian Noise }\end{array}$ & $\begin{array}{l}\text { PD using Fuzzy Threshold- } \\
\text { ing under Gaussian Noise }\end{array}$ & $\begin{array}{l}\text { PD using Fuzzy Thresholding } \\
\text { under Non-Gaussian Noise }\end{array}$ \\
\hline-15 & 0.04 & 0.05 & 0.15 & 0.12 \\
-10 & 0.06 & 0.06 & 0.18 & 0.15 \\
\hline
\end{tabular}

Realizing Eqn (10-17) in MATLAB and by appropriately choosing the membership functions and the fuzzy rule base in the fuzzy inference system the proposed adaptive thresholding using fuzzy logic method for ED under Laplacian noise, provides reliable detection compared to the other methods when the noise uncertainty increases. Another interesting observation drawn is on the improvement in signal detection in the low SNR regimes.

From Figure 7 it is observed that for the conditions in the existing literature on sensing under Laplacian noise ${ }^{(19)}$, the fuzzy thresholding provides $20 \%$ increase in the detection, at false alarm rate $0.01,1000$ samples and $\rho$ of $0.1 \mathrm{~dB}$. Table 3 summarizes the value of probability of detection obtained in Gaussian and non-Gaussian environments using both the thresholding schemes. It is observed that the fuzzy thresholding method provides increased detection accuracy of around $20 \%$ compared to the single thresholding scheme.

Kurtosis refers to the size of the tails on a distribution. The tails of a distribution quantify the number of events which have appeared that are outside the standard range. If the distributions of event outcomes have lots of occurrences of outlier results, this causes heavy tails on the bell-shaped distribution curve. This is referred to as excess kurtosis. A kurtosis of 3 is termed as mesokurtic. Standard normal distributions are mesokurtic. If the kurtosis is $>3$ it can be visualized as a thin bell-shaped curve with a high peak termed as leptokurtic. One of the most well-known leptokurtic distributions is Student's t distribution, Laplace distribution. When kurtosis $<3$ is recognized as platykurtic with broad peak and thick tails. All uniform distributions are platykurtic.

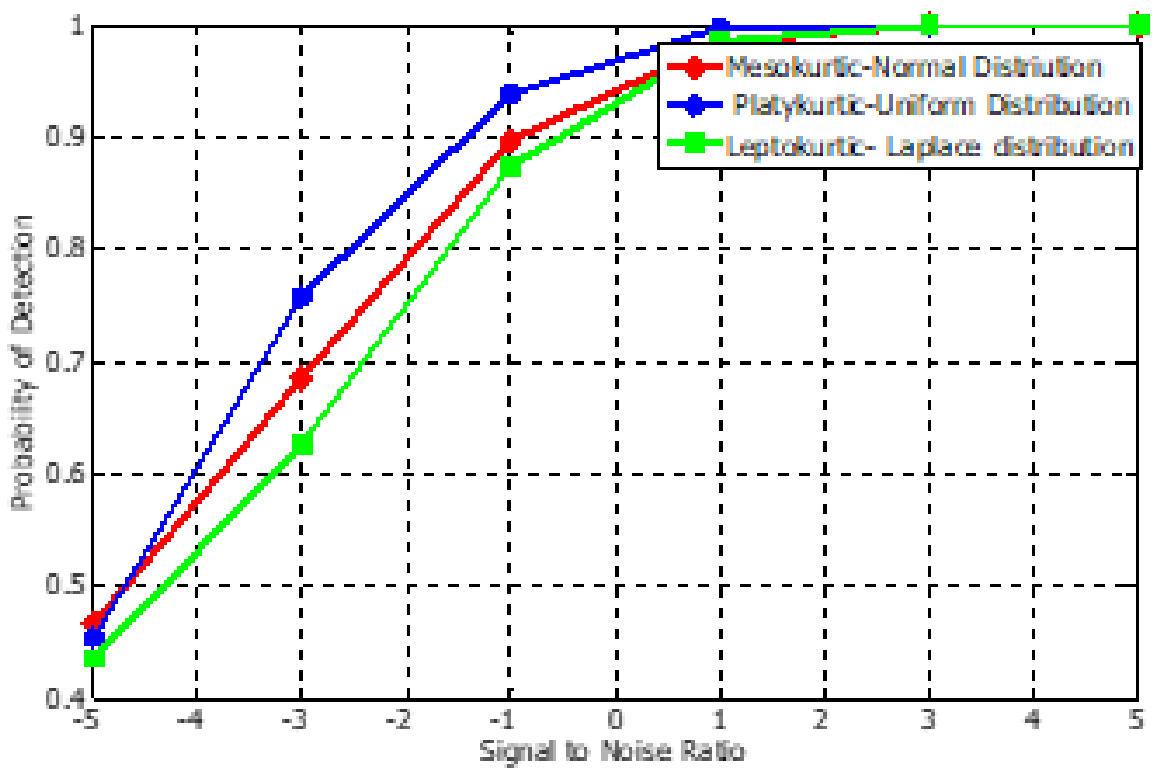

Fig 9. Effect of Kurtos is on primary user detection

The detection probability achieved for the mesokurtic, platykurtic and leptokurtic distributions are plotted in Figure 9. As the kurtosis increases, the detection probability decreases. This is because the platykurtic distribution produces fewer outliers and less extremes than the normal distribution. 
In the leptokurtic distribution, like the Laplace distribution, the tails approach asymptotically to zero more slowly than a Gaussian. Hence the number of outliers are more than the normal distribution. Thus, the uniform distribution with fewer numbers of outlier's shows higher PU signal detection compared to normal and Laplace distributions.

\section{Conclusion}

In this paper, some typical impairments in PU detection, such as the effect of a non-Gaussian noise and noise uncertainty are investigated. Majority of spectrum sensing schemes are focused on primary user detection in Gaussian noise. But in practical wireless communication scenarios the noise usually has a heavy tailed nature. The Laplacian noise is an important non-Gaussian noise distributions and hence it is frequently used in engineering studies. This work focuses on primary user signal detection in Laplacian noise. Through Monte Carlo simulations it is observed that a non-Gaussian noise noticeably affects the performance of ED. Also a fractional change in noise uncertainty degrades the performance of energy detector. Hence to combat the above degradation in energy detector's performance the spectrum sensing algorithm using adaptive fuzzy thresholding which was primarily studied under Gaussian noise is extended under a non-Gaussian noise environment. Through simulations it is shown that by appropriately choosing the membership functions and the fuzzy rule base in the fuzzy inference system the proposed adaptive thresholding using fuzzy logic method for Laplacian energy detector provides reliable detection. The effect of the probability of detection due to the characterization of the skewness and kurtosis are also studied. Results show that as the kurtosis increases, the tails are heavier and it degrades the PU detection. The study limits to the use of single antenna in the sensing process. Hence the effect of using multiple antennas in the sensing process and in fading scenarios can be considered for future investigation in a non-Gaussian noise environment.

\section{References}

1) Kumar A, Thakur P, Pandit S, Singh G. Threshold selection and cooperation in fading environment of cognitive radio network: Consequences on spectrum sensing and throughput. AEU - International Journal of Electronics and Communications. 2020;117:153101. Available from: https://dx.doi.org/10.1016/j. aeue.2020.153101.

2) Sarala B, Devi SR, Sheela JJJ. Spectrum energy detection in cognitive radio networks based on a novel adaptive threshold energy detection method. Computer Communications. 2020;152:1-7. Available from: https://dx.doi.org/10.1016/j.comcom.2019.12.058.

3) Yu S, Liu J, Wang J, Ullah I. Adaptive Double-Threshold Cooperative Spectrum Sensing Algorithm Based on History Energy Detection. Wireless Communications and Mobile Computing. 2020;2020:1-12. Available from: https://dx.doi.org/10.1155/2020/4794136.

4) Mahendru G, Shukla AK, Banerjee P, Patnaik LM. Adaptive Double Threshold Based Spectrum Sensing to Overcome Sensing Failure in Presence of Noise Uncertainty. 2019 6th International Conference on Signal Processing and Integrated Networks (SPIN). 2019;p. 466-471. Available from: https://doi.org/10.1109/SPIN.2019.8711570.

5) Salahdine F, Kaabouch N, Ghazi HE. Techniques for dealing with uncertainty in cognitive radio networks. 2017 IEEE 7th Annual Computing and Communication Workshop and Conference (CCWC). 2017;p. 1-6. Available from: https://doi.org/10.1109/CCWC.2017.7868352.

6) Yang T, Wu Y, Li L, Xu W, Tan W. A Two-Step Cooperative Energy Detection Algorithm Robust to Noise Uncertainty. Wireless Communications and Mobile Computing. 2019;2019:1-10. Available from: https://dx.doi.org/10.1155/2019/3912784.

7) Reddy DN, Ravinder Y. Novel receiver diversity combining methods for spectrum sensing using meta-analytic approach based on p-values. J Eng Sc Tech. 2018;13(9):2883-2897.

8) Gao R, Qi P, Zhang Z. Frequency domain goodness of fit test based spectrum sensing method with dynamically varying noise. China Communications. 2020;17(12):172-179. Available from: https://dx.doi.org/10.23919/jcc.2020.12.012.

9) Ahuja B, Kaur G. Two-Stage Spectrum Sensing Using Fuzzy Logic for Cognitive Radio Networks. Proceedings of the National Academy of Sciences, India Section A: Physical Sciences. 2020;90(3):515-525. Available from: https://dx.doi.org/10.1007/s40010-019-00595-7.

10) Bharatula S, Murthy BS. Innovative Fuzzy-Based Spectrum Sensing Technique for Noisy Conditions. In: Lecture Notes in Electrical Engineering. Springer Singapore. 2021;p. 735-750. doi:10.1007/978-981-15-9019-1_64.

11) Mabrook MM, Taha HA, Hussein AI. Cooperative spectrum sensing optimization based adaptive neuro-fuzzy inference system (ANFIS) in cognitive radio networks. Journal of Ambient Intelligence and Humanized Computing. 2020;5:1-2. Available from: https://dx.doi.org/10.1007/s12652-020-02121-9.

12) Sinha K, Trivedi YN. Spectrum Sensing Based on Dynamic Primary User with Additive Laplacian Noise in Cognitive Radio. In: Lecture Notes of the Institute for Computer Sciences, Social Informatics and Telecommunications Engineering. Springer International Publishing. 2021;p. 16-28. Available from: http://dx.doi.org/10.1007/978-3-030-73423-7_2.

13) Gao R, Jing F, Wang J, Zhang J, Zhang Y. A New Spectrum Sensing Method with Low SNR under Laplace Noise. 2020 IEEE 3rd International Conference of Safe Production and Informatization (IICSPI). 2020;p. 291-297. Available from: https://doi.org/10.1109/IICSPI51290.2020.9332346.

14) Gao R, Qi P, Zhang Z. Performance analysis of spectrum sensing schemes based on energy detector in generalized Gaussian noise. Signal Processing. 2021;181:107893. Available from: https://dx.doi.org/10.1016/j.sigpro.2020.107893.

15) Tan F, Song X, Leung C, Cheng J. Collaborative Spectrum Sensing in a Cognitive Radio System with Laplacian Noise. IEEE Communications Letters. 2012;16(10):1691-1694. Available from: https://dx.doi.org/10.1109/lcomm.2012.080312.120517.

16) Ahuja B, Kaur G. Two-stage spectrum sensing using fuzzy logic for cognitive radio networks. Proceedings of the National Academy of Sciences, India Section A: Physical Sciences. 2020;90:515-540.

17) Celikoglu A, Tirnakli U. Skewness and kurtosis analysis for non-Gaussian distributions. Physica A: Statistical Mechanics and its Applications. 2018;499:325334. Available from: https://dx.doi.org/10.1016/j.physa.2018.02.035.

18) Aryal GR. Study of laplace and related probability distributions and their applications. . Available from: https://scholarcommons.usf.edu/etd/2443/. 
19) Gao R, Li Z, Li H, Ai B. Absolute Value Cumulating Based Spectrum Sensing with Laplacian Noise in Cognitive Radio Networks. Wireless Personal Communications. 2015;83(2):1387-1404. Available from: https://dx.doi.org/10.1007/s11277-015-2457-4.

20) Tandra R, Sahai A. SNR Walls for Signal Detection. IEEE Journal of Selected Topics in Signal Processing. 2008;2(1):4-17. Available from: https: //dx.doi.org/10.1109/jstsp.2007.914879. 